\title{
Relativistic covariance of quasipotential equations
}

\author{
V. Pascalutsa and J.A. Tjon \\ Institute for Theoretical Physics, University of Utrecht, 3584 CC Utrecht, The Netherlands
}

(November 3, 1997)

\begin{abstract}
We argue that most of the relativistic 3-D (quasipotential) equations used in hadron physics are inconsistent with the discrete symmetries like charge conjugation and CPT, yielding an incorrect Lorentz structure for the calculated Green's functions. An exception to this is the equal time approximation to the Bethe-Salpeter equation. We present a covariant quasipotential model for the pion-nucleon interaction based on hadronic degrees of freedom and satisfying the full covariance.
\end{abstract}

PACS number(s): 11.10.St, 11.30.Er, 21.45.+v, 13.75.Gx

Keywords: Relativistic quasipotential equations; Discrete Lorentz symmetries; Pion-nucleon interaction

In recent years there has been a considerable interest to formulate 3-dimensional (3-D) dynamical equations for the $\pi N$ system satisfying relativistic covariance [1,2]. Within a relativistic field theory framework the Bethe-Salpeter (BS) equation, see Fig. 1, can conveniently be used to carry out a manifestly covariant reduction to a 3-D quasipotential (QP) equation [3 [7. This reduction involves an assumption about the singularities of the BS kernel after which the integration over the 0 -th component (time or energy) can easily be done explicitly. There is, however, no unique scheme for the choice of the QP equation. The covariant reduction can be done in infinitely many different ways. Certainly, one would like to restrict the choice not only by fitting to experimental data, some restrictions can come from various symmetries and consistency requirements, such as the correct low-energy limit, the correct one-body limit of the equation [5, etc. In this Letter we discuss the constraints due to discrete Lorentz symmetries. Within a framework satisfying these symmetries, we construct a relativistic dynamical model of the $\pi N$ interaction.

In considering existing relativistic 3-D equations, we find that they in general do not have the correct Lorentz structure. For example, the calculated self-energy of a spin-1/2 particle does not have the following form (required by the full Lorentz covariance),

$$
\Sigma(\not P)=\not P A\left(P^{2}\right)+B\left(P^{2}\right),
$$

where $A$ and $B$ are scalar functions of the invariant $P^{2}$ only. At first this result is rather surprising, naively we would expect form (1) as a natural outcome of any covariant formalism.

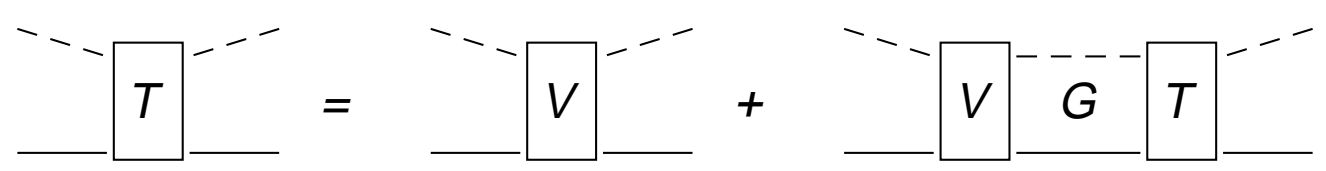

FIG. 1. Bethe-Salpeter equation for the $\pi N$ scattering amplitude 
The violation of the Lorentz structure is intimately related to the way the singularities of the BS kernel are treated in doing the QP reduction. To see this, let us consider a simple example of the scalar self-energy, given by

$$
\Sigma_{S}\left(P^{2}\right)=i \int \frac{\mathrm{d}^{4} k}{(2 \pi)^{4}} \frac{\Phi\left(k^{2}, P^{2}, P \cdot k\right)}{\left[\left(\frac{1}{2} P-k\right)^{2}-m^{2}-i \epsilon\right]\left[\left(\frac{1}{2} P+k\right)^{2}-m^{2}-i \epsilon\right]},
$$

where $P$ is the relevant 4-vector, $\Phi$ is some "interaction function" which corresponds to the product of the two vertex functions, and which may also have some particle propagation poles.

We immediately see that Eq. (2) is a function of $P^{2}$ only: a sign change of $P$ can be absorbed by a change of the loop variable $k$ in Eq. (2) to $-k$. In a QP description this substitution in general cannot be applied in view of the constraint in $k_{0}$. We can readily convince ourself that in that case $\Sigma_{S}$ is in general not an even function of $P_{0}$ anymore. Consider the poles of the integrand of Eq. (2) in the complex $k_{0}$ plane. There are four poles (two in the upper and two in the lower half-plane) coming from the propagators in the 2-particle Green function:

$$
k_{0}= \pm \frac{1}{2} P_{0}-\sqrt{m^{2}+\left(\frac{1}{2} \vec{P} \mp \vec{k}\right)^{2}}+i \epsilon \text { and } k_{0}= \pm \frac{1}{2} P_{0}+\sqrt{m^{2}+\left(\frac{1}{2} \vec{P} \mp \vec{k}\right)^{2}}-i \epsilon .
$$

We see that with a sign change of $P_{0}$ and $k_{0}$ the poles of the upper half-plane are interchanged with the poles of the lower half-plane. The same symmetry exists for the singularities of $\Phi$. Therefore, in order for $\Sigma_{S}$ to be even in $P_{0}$ the integration over $k_{0}$ must be independent of the choice of the half-plane where the contour is closed. In doing a $3-\mathrm{D}$ reduction, however, one usually neglects the contribution of certain poles, hence the result becomes dependent on the way the contour is closed, and $\Sigma_{S}$ is not anymore an even function of $P_{0}$ and consequently it cannot depend only on $P^{2}$. In this sense the Lorentz structure of the self-energy is violated.

From this example we can see, that in order to simplify the singularity structure, preserving the full Lorentz covariance, one should first remove the 'unwanted' poles, and not merely neglect them in doing the reduction. The so-called equal-time (ET) approximation [7] precisely provides a procedure for removing the singularities in the $k_{0}$ variable. Using the ET prescription we thus may get rid of as many poles as we like (usually these are poles of the vertex functions) and then perform the $k_{0}$ integration exactly. For example, in the case of the scalar self-energy, the standard ET procedure would be to remove the poles of $\Phi$ by introducing a quasipotential approximation to the interaction function:

$$
\Phi_{Q P}\left(\tilde{k}^{2}, P^{2}\right)=\Phi\left(\tilde{k}^{2}, P^{2}, P \cdot \tilde{k}\right)
$$

with $\tilde{k}=k-P(P \cdot k) / P^{2}$. The self-energy becomes in this approximation

$$
\Sigma_{S}\left(P^{2}\right) \stackrel{E T}{=} i \int \frac{\mathrm{d}^{4} k}{(2 \pi)^{4}} \frac{\Phi_{Q P}\left(\tilde{k}^{2}, P^{2}\right)}{\left[\left(\frac{1}{2} P-k\right)^{2}-m^{2}-i \epsilon\right]\left[\left(\frac{1}{2} P+k\right)^{2}-m^{2}-i \epsilon\right]}
$$

Due to the manifestly covariant form of Eq. (4),$\Sigma_{S}$ is clearly a function of $P^{2}$ only. In the c.m. frame $(\vec{P}=0)$ Eq. (价 has a particularly simple form. Then the interaction function $\Phi_{Q P}\left(-\vec{k}^{2}, P^{2}\right)$ is independent of $k_{0}$ and hence the only remaining singularities of Eq. (4) in the $k_{0}$ plane are the poles of the 2-particle Green function. Working out the integral over $k_{0}$ leads to the 3 -D formulation.

Obviously, similar arguments apply for the spin-1/2 particle self-energy. Consider the dressed nucleon propagator given by

$$
S(\not P)=[\not P-m-\Sigma(\not P)-i \epsilon]^{-1},
$$


where $\Sigma(\mathbb{P})$ is the self-energy. For simplicity we work in the c.m. frame, where $P=\left(P_{0}, \overrightarrow{0}\right)$. In this frame the Dirac structure of the self-energy can simply be represented as

$$
\Sigma\left(P_{0}\right)=\Sigma_{+}\left(P_{0}\right) \gamma_{+}+\Sigma_{-}\left(P_{0}\right) \gamma_{-}
$$

where $\gamma_{+}=\frac{1}{2}\left(I+\gamma_{0}\right), \gamma_{-}=\frac{1}{2}\left(I-\gamma_{0}\right)$. A similar decomposition holds for the propagator,

$$
S\left(P_{0}\right)=S^{(+)}\left(P_{0}\right) \gamma_{+}+S^{(-)}\left(P_{0}\right) \gamma_{-}
$$

with $S^{( \pm)}\left(P_{0}\right)= \pm\left[P_{0} \pm\left(-m-\Sigma_{ \pm}\left(P_{0}\right)+i \epsilon\right)\right]^{-1}$. Obviously, $S^{(+)}$corresponds to the positive and $S^{(-)}$to the negative energy-state propagation.

It is easy to see that, if the self-energy can be written in the general covariant form (11), then the following identity holds,

$$
\Sigma_{r}\left(P_{0}\right)=\Sigma_{-r}\left(-P_{0}\right), \quad r= \pm 1
$$

and vise versa (in the c.m. frame). Having made this connection, we may easily test numerically Eq. (8) to see whether the form (1) is preserved in a given dynamical model.

As was briefly reported [8], we develop a unitary dynamical model for the $\pi N$ interaction based on the solution of the BS equation in the ET approximation. For the 'driving force' we take the tree-level $\rho$-, $\sigma$ meson and $N, N^{*}(1470), \Delta(1232)$-baryon exchanges, see Fig. 2. The $\Delta$ and $N^{*}$ are widthless in the driving force, their one-pion-nucleon decay width is then generated dynamically in the calculation. In contrast to Ref. [2] no static approximation has been made. Note that, although the potential is crossing symmetric, the kernel of the equation and thus the resulting amplitude is not.

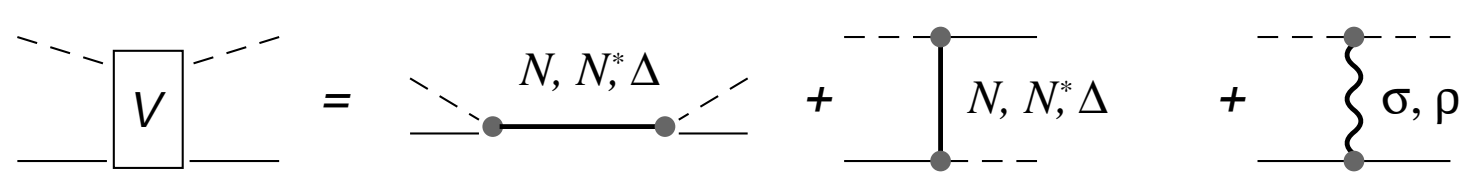

FIG. 2. The driving force in the $\pi N$ model.

The bare vertices and propagators are taken from an effective Lagrangian of the meson and the isobar fields. We allow the pion field to be coupled only through a derivative coupling, which directly provides the correct low-energy limit, at least at the tree level. The rescattering can in principle violate the low-energy limit (in our model this may come due to the lack of crossing symmetry). We have checked numerically that these violations are small in our model by running a similar test as in Ref. [1]. For the propagator of the $\Delta$ we use the Rarita-Schwinger propagator, and the $\pi N \Delta$ coupling is taken to be of the general structure determined by a coupling constant $f_{\pi N \Delta}$ and an off-shell parameter $z$, cf. Ref. [9]. The $\rho$ exchange generates the isovector-vector current. The strength of its coupling is rather close to that determined by the Kawarabayashi-Suzuki relation ( $g_{\pi N N}$ which we use would imply $g_{\rho N N} g_{\rho \pi \pi} / 4 \pi \approx 2.8$ ). The $\rho$ thus mainly plays the role of the $\pi N$ contact term required in the non-linear realizations of chiral symmetry. The $\sigma$ meson is included so as to simulate the isoscalar-scalar contribution of the correlated two-pion exchange. Therefore, for the $\sigma \pi \pi$ coupling constant we take the sign determined in Ref. [10], and we find that this choice is preferable for the proper description of the phase-shifts.

For each particle we have used a form factor depending on the 4-momentum squared of the particle. For a meson we take the one-boson-exchange form factor, and for a baryon we use the form factor of Pearce 
and Jennings [1] (with $n_{\alpha}=2$ ). The corresponding cutoff masses and other model parameters were fitted to the $\pi N$ phase-shifts. Their values are given in Table 1. Our fit to the $\mathrm{S}$ - and P-waves up to $400 \mathrm{MeV}$ pion kinetic energy is presented in Fig. 3 .
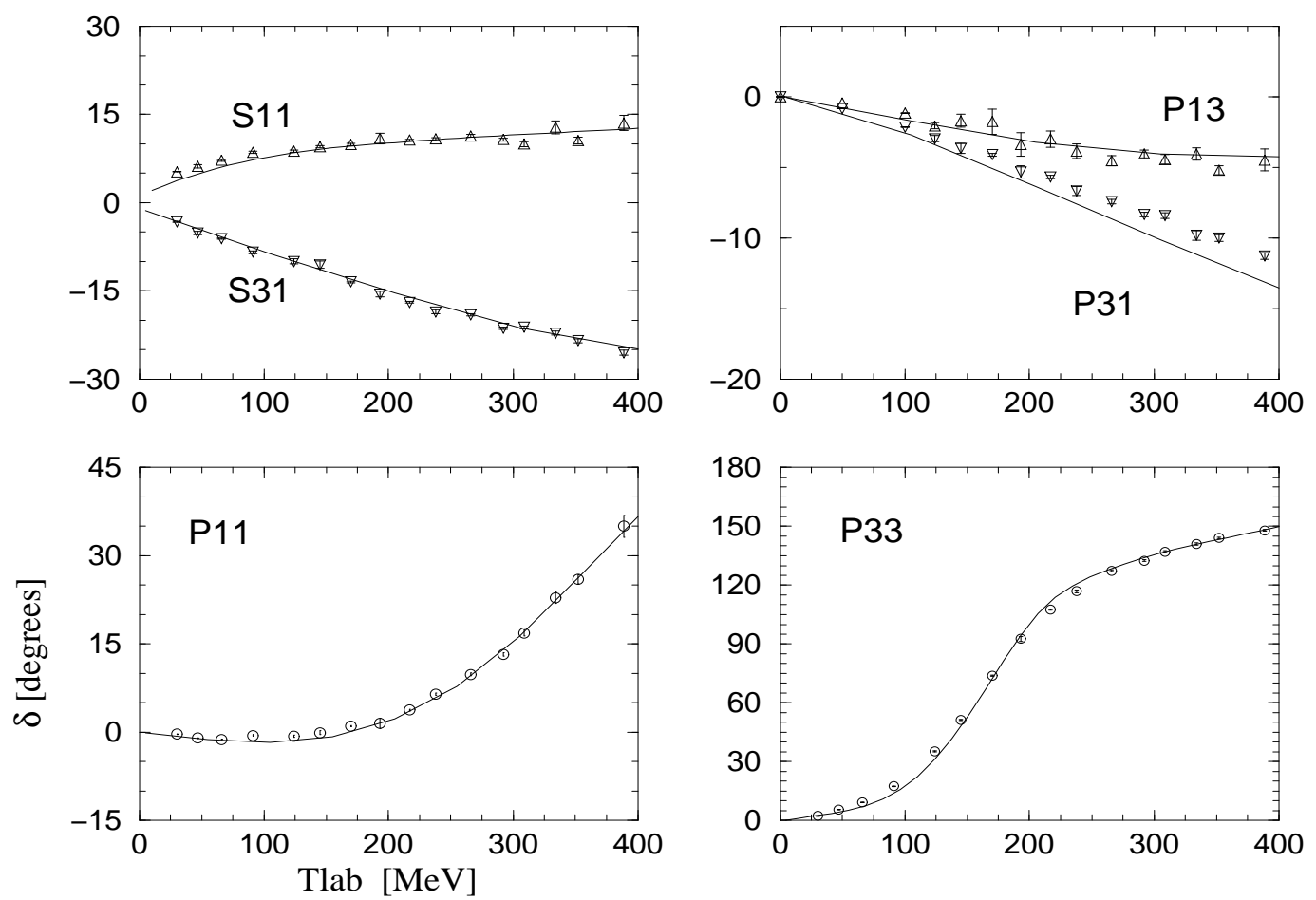

FIG. 3. The S- and P-wave $\pi N$ phase-shifts. Solid lines represent our calculation, data points are from the SM95 partial-wave analysis [1].

\begin{tabular}{cc}
\hline \hline coupling constants & masses $[\mathrm{GeV}]$ \\
\hline$g_{\pi N N}^{2} / 4 \pi=13.5$ & $\Lambda_{N}=1.5, \Lambda_{\pi}=1.5$ \\
$g_{\pi N N^{*}}^{2} / 4 \pi=0.9$ & $\Lambda_{N^{*}}=1.9, m_{N^{*}}=1.54$ \\
$f_{\pi N \Delta}^{2} / 4 \pi=0.33, z=-0.2$ & $\Lambda_{\Delta}=1.4, m_{\Delta}=1.24$ \\
$g_{\sigma N N} g_{\sigma \pi \pi} / 4 \pi=0.1$ & $\Lambda_{\sigma}=1.1, m_{\sigma}=0.55$ \\
$g_{\rho N N} g_{\rho \pi \pi} / 4 \pi=2.5, \kappa_{\rho}=3.7$ & $\Lambda_{\rho}=1.1, m_{\rho}=0.77$ \\
\hline \hline
\end{tabular}

TABLE I. The model parameters which were ajusted to reproduce the $\pi N$ phase-shifts. Only the physical (renormalized) values of parameters are given. 
We shall now corroborate the issue of the full relativistic covariance in an explicit calculation of the selfenergy contribution in our model versus the models based on other relativistic QP equations for $\pi N$ system [1.2].

The field theoretical nucleon self-energy in the models based on the BS equation can be written, after the partial-wave decomposition, as follows,

$$
\Sigma_{r}\left(P_{0}\right)=\frac{i}{\pi} \int_{-\infty}^{\infty} \frac{\mathrm{d} k_{0}}{2 \pi} \int_{0}^{\infty} \frac{\mathrm{dk}}{2 \pi} \mathrm{k}^{2} \sum_{\rho} G^{(\rho)}\left(\mathrm{k}, k_{0} ; P_{0}\right) \Phi_{r}^{(\rho)}\left(\mathrm{k}, k_{0} ; P_{0}\right)
$$

where $G^{(\rho)}$ is the $\pi N$ propagator and the interaction function $\Phi_{r}^{(\rho)}$ represents the $\pi N N$ vertex contributions with $\rho$ characterizing the $\rho$-spin [7] of the intermediate state and $r$ characterizing parity. Furthermore, the integration variable $k_{0}$ is the relative-energy variable, defined as

$$
k_{0}=\left(p_{\mathrm{N} 0} \hat{\omega}-p_{\pi 0} \hat{E}\right) / P_{0}
$$

where $p_{\text {No }}\left(p_{\pi 0}\right)$ is the 0 -th component of the nucleon (pion) 4-momentum, and $\hat{\omega}=\left(P_{0}^{2}-m^{2}+m_{\pi}^{2}\right) / 2 P_{0}, \hat{E}=$ $\left(P_{0}^{2}+m^{2}-m_{\pi}^{2}\right) / 2 P_{0}$. Then $G^{(\rho)}$ has the following explicit form,

$$
G^{(\rho)}\left(k, k_{0} ; P_{0}\right)=\rho\left\{\left(\hat{E}+k_{0}-\rho E_{k}+i \epsilon\right)\left[\left(\hat{\omega}-k_{0}\right)^{2}-\omega_{k}^{2}-i \epsilon\right]\right\}^{-1},
$$

which clearly indicates the position of its poles in $k_{0}$ plane:

$$
k_{0}^{(\pi \pm)}=\hat{\omega} \mp\left(\omega_{k}+i \epsilon\right), \quad k_{0}^{(\mathrm{N} \pm)}=-\hat{E} \pm\left(E_{k}-i \epsilon\right),
$$

with $E_{k}=\sqrt{m^{2}+\mathrm{k}^{2}}, \omega_{k}=\sqrt{m_{\pi}^{2}+\mathrm{k}^{2}}$. Also, $\Phi_{r}^{(\rho)}$ has poles associated with those of the potential and the form factors

In the ET approximation we have, instead of Eq. (9),

$$
\Sigma_{r}\left(P_{0}\right) \stackrel{E T}{=} \frac{1}{\pi} \int_{0}^{\infty} \frac{\mathrm{d} \mathrm{k}}{2 \pi} \mathrm{k}^{2} \sum_{\rho} G_{\mathrm{ET}}^{(\rho)}\left(\mathrm{k} ; P_{0}\right) \Phi_{r}^{(\rho)}\left(\mathrm{k}, 0 ; P_{0}\right),
$$

where

$$
G_{\mathrm{ET}}^{(\rho)}\left(\mathrm{k} ; P_{0}\right)=i \int_{-\infty}^{\infty} \frac{\mathrm{d} k_{0}}{2 \pi} G^{(\rho)}\left(\mathrm{k}, k_{0} ; P_{0}\right)=-\rho\left\{2 \omega_{k}\left(-\rho P_{0}+E_{k}+\omega_{k}+i \epsilon\right)\right\}^{-1} .
$$

In Fig. 1 we plot the results of the calculation of the nucleon self-energy to one $\pi N$ loop, using the pseudovector coupling and the form factors described above. As Fig. 1 shows, within the ET approximation the results for $\Sigma_{+}\left(P_{0}\right)$ and $\Sigma_{-}\left(-P_{0}\right)$ are exactly the same. As an example of a QP prescription which violates the full covariance we may consider the spectator (or Gross) equation, where one of the particles in the loop is on its mass shell. Formally, in doing the $k_{0}$ integral the contribution of only one pole is taken into account: it can be either $k_{0}^{(\pi+)}$ in Eq. (12) - the pion spectator, or $k_{0}^{(N+)}$ - the nucleon spectator. The "pion spectator" was preferred by Gross and Surya [2], and we have also used this choice to carry out the

\footnotetext{
${ }^{1}$ In the lowest order the r.h.s of Eq. (9) is simply the one $\pi N$ loop with $\Phi_{r}^{(\rho)}$ the product of the two bare $\pi N N$ vertices.
} 
calculation presented in Fig. (4. The figure clearly shows that the desirable identity Eq. (8) is not satisfied for the spectator model. Of course, the size of the violation is model-dependent, but is unlikely to vanish for all energies in any model. Similar violations arise in the equations used by Pearce and Jennings [1]. In particular, their choice of the Blankenbecler-Sugar propagator has this problem due to the presence of the positive-energy projection operator, which, again, leaves out the contribution of the negative-energy pole. In contrast, the ET approximation satisfies the identity Eq. (8) exactly, thus preserving the Lorentz structure given in Eq. (11).

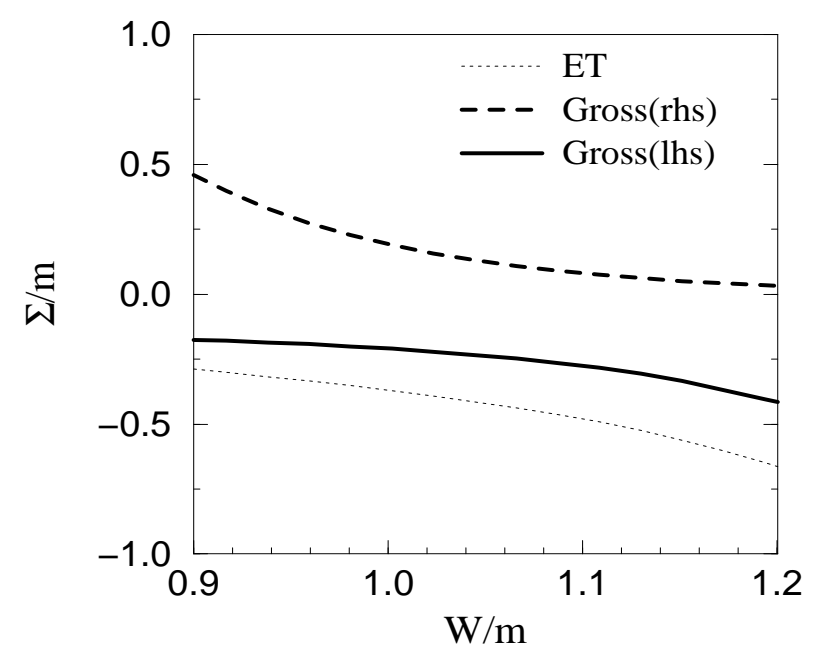

FIG. 4. The r.h.s. and 1.h.s. of Eq. (\&) for the one $\pi N$-loop using the Gross prescription (pion spectator model). For the ET approximation they are found to be identical, given by the dotted line. Both the self-energy and the energy, $W=P_{0}$ are in units of the nucleon mass.

Note that all of the considered models [1,2,8] maintain the exact two-particle (elastic) unitarity. They all thus give the following expression for the imaginary part of the nucleon self-energy,

$$
\operatorname{Im} \Sigma_{r}\left(P_{0}\right)=-\frac{q \hat{E}}{4 \pi P_{0}} \Phi_{r}^{(+)}\left(q, 0 ; P_{0}\right) \theta\left(q^{2}\right)
$$

where $q$ the center-of-mass momentum, $\theta$ is the step function. In employing the ET prescription, we could have for example tried to remove one of the poles of the $\pi N$ propagator. However, in doing so we loose the two-particle unitarity of the equation. Thus the condition of elastic unitarity and full Lorentz covariance clearly restricts the form of allowed ET approximation.

Since s-channel singularities are present in our force model, we apply a renormalization procedure. Considering for instance the dressed nucleon propagator (5), we see that the positive and negative bare mass singularities in Eq. (7) are shifted equally in view of Eq. (8), yielding poles in the dressed propagator at $P_{0}= \pm m, m$ being the physical nucleon mass. Consequently, a standard renormalization constant $Z_{2}$ which transforms like a scalar can be used. If Eq. (8) does not hold, such a renormalization procedure breaks down. In particular, it would require non-covariant subtraction constants.

Hence, we may conclude that the violation of identity (8) leads to the difference in renormalization of the positive and negative energy states. This obviously signals the violation of CPT symmetry. The noncovariance found in some QP prescriptions is indeed related to the violation of certain discrete Lorentz symmetries. One can show that invariance under charge conjugation, parity and time reversal implies the following transformation properties for the spin- $1 / 2$ particle self-energy, 


$$
\begin{aligned}
& C: C \Sigma\left(p_{0}, \vec{p}\right) C^{-1}=\Sigma^{T}\left(-p_{0},-\vec{p}\right), \\
& P: \gamma_{0} \Sigma\left(p_{0}, \vec{p}\right) \gamma_{0}=\Sigma\left(p_{0},-\vec{p}\right), \\
& T: \gamma_{0} C^{-1} \gamma_{5} \Sigma\left(p_{0}, \vec{p}\right) \gamma_{5} C \gamma_{0}=\Sigma^{T}\left(p_{0},-\vec{p}\right), \\
& C P T: \gamma_{5} \Sigma\left(p_{0}, \vec{p}\right) \gamma_{5}=\Sigma\left(-p_{0},-\vec{p}\right),
\end{aligned}
$$

where superscript $T$ means transposition in the $\gamma$-matrix space and $C$ is the charge conjugation matrix (note that here $\mathrm{CPT}$ and $\mathrm{C}$ imply essentially the same condition, since $\gamma_{5}$ and the $C$-matrix act very similar on $\gamma_{\mu}: \gamma_{5} \gamma_{\mu} \gamma_{5}=-\gamma_{\mu}, C \gamma_{\mu} C^{-1}=-\gamma_{\mu}^{T}$ ). From this we see that the violation of Eq. (8) appears as the violation of the charge conjugation and, as a consequence, CPT symmetry.

In conclusion, we have presented a relativistic unitary model for $\pi N$ interaction based on the ET type of formulation which is consistent also with the discrete Lorentz symmetries, in contrast to the models based on other relativistic 3-D formulations. The violations of the discrete Lorentz symmetries, such as charge conjugation and CPT, occur in the most of the 3-D formulations because of the difference in the treatment of positive and negative energy states. These violations are reflected in the violation of the Lorentz structure, i.e. dependences on not only the standard covariants. Therefore, in these cases, one should be careful to use covariant arguments to construct the transformation properties of the calculated amplitudes, which would be needed when we want to incorporate the basic interaction in more particle systems. One should also be careful in exploiting the usual Ward identities, low-energy theorems, power-counting arguments.

[1] B.C. Pearce and B.K. Jennings, Nucl. Phys. A528 (1991) 655.

[2] F. Gross and Y. Surya, Phys. Rev. C 47 (1993) 703; ibid. 53 (1996) 2422.

[3] A.A. Logunov and A.N. Tavkhelidze, Nuovo Cim. 29 (1963) 380;

R. Blankenbecler and R. Sugar, Phys. Rev. 142 (1966) 1051.

[4] I.T. Todorov, Quasipotential Approach to the Two-Body Problem in Quantum Field Theory, in: Properties of the Fundamental Interactions, Vol. 9, part C, ed.A. Zichichi (Bologna, 1973) p. 953, and references therein.

[5] F. Gross, Phys. Rev. 186 (1969) 1448; Phys. Rev. C 26 (1982) 2203.

[6] V.B. Mandelzweig and S.J. Wallace, Phys. Lett. B197 (1987) 469;

S.J. Wallace and V.B. Mandelzweig, Nucl. Phys. A503 (1989) 673.

[7] J.A. Tjon, Hadronic Physics with multi-GeV Electrons, in: Les Houches Series (New Science Publishers, New York, 1990) page 89.

[8] V. Pascalutsa and J.A. Tjon, in: Proc. XV Int. Conf. on Few-Body Problems in Physics (Groningen, 1997), to be published in Nucl. Phys. A, nucl-th/9709017.

[9] L.M. Nath, B. Etemadi, J.D. Kimel, Phys. Rev. D 3 (1971) 2153.

[10] C. Schültz, J.W. Durzo, K. Holinde and J. Speth, Phys. Rev. C 49 (1994) 2671; ibid. 51 (1995) 1374.

[11] R.A. Arndt, I. Strakovsky, R.L. Workman, Phys. Rev. C 52 (1995) 2120. 